\title{
SISTEMAS PRODUCTIVOS LOCALES: ESTRATEGIAS EMPRESARIALES PARA EL DESARROLLO*
}

\author{
Recibido: 28 de octubre de 2013 • Aprobado: 15 de mayo de 2014 \\ Jaime Alberto Rendón Acevedo** \\ Jesús David Forero Muñoz ${ }^{* * *}$
}

RESUMEN

El objetivo de este artículo es proponer los sistemas productivos locales como una estrategia de industrialización y desarrollo. Para cumplir con tal objetivo se presenta qué son teóricamente los sistemas productivos locales y se expone por qué son importantes en el desarrollo. Dos conclusiones principales: la primera es que los sistemas productivos locales son una forma de aglomeración empresarial adecuada para países como Colombia, cuyo censo empresarial se compone casi en su totalidad por micro y pequeñas empresas; y la segunda es que incentivan el desarrollo de las comunidades, al pensar el desarrollo desde el territorio mismo.

\section{PALABRAS CLAVE}

Desarrollo local, cadena productiva, sistema productivo local, distritos industriales.

CLASIFICACIÓN JEL

L16, R11, R39

\section{CONTENIDO}

Introducción; 1. Sistemas productivos locales; 2. Economías externas y la configuración de los Sistemas Productivos Locales; 3. Conclusiones; Bibliografía.

\footnotetext{
Este artículo de investigación es producto del proyecto denominado "Sistemas productivos y responsabilidad social en Bogotá", el cual fue financiado por la Vicerrectoría de Investigaciones y Transferencia de la Universidad de La Salle, Bogotá, Colombia.

"* Economista, Facultad de Ciencias Económicas, Universidad de Antioquia, Colombia. Doctor en Economía Internacional y Desarrollo, Universidad Complutense de Madrid (España). Integrante del grupo interdisciplinario de investigación en Desarrollo, Estructuras Económicas, Políticas Públicas y Gestión (Categoría D en Colciencias). Facultad de Ciencias Económicas y Sociales, Universidad de La Salle. Director Centro de Estudios en Desarrollo y Territorio (CEDT), Correo electrónico: jrendon@unisalle.edu.co.

***Economista, Universidad de La Salle, Bogotá, Colombia. Pasante de investigación del CEDT y estudiante de la Maestría en Estudios y Gestión del Desarrollo, Universidad de la Salle, Bogotá, Colombia. Correo electrónico: jforero46@unisalle.edu.co.
} 


\section{LOCAL PRODUCTIVE SYSTEMS: CORPORATE STRATEGIES FOR DEVELOPMENT ABSTRACT}

The objective of this paper is to propose local productive systems as an industrial and development strategy. To comply with such objective, the theory of the local productive systems is presented and the reason of their importance in development is exposed. Two main conclusions arose: the first one is that local productive systems are a way of corporate agglomeration that is adequate for countries such as Colombia, which corporate census is mainly composed by small and middle sized businesses; and the second one is that they do work as incentives for community development when thinking on development from the territory.

\section{KEY WORDS}

Local development, productive chain, local productive system, industrial districs.

Desarrollo local, cadena productiva, sistema productivo local, distritos industriales.

JEL CLASSIFICATION

L16, R11, R39

\section{CONTENT}

Introduction; 1. Local productive systems; 2. External economies and the configuration of Local Productive Systems; 3. Conclusions; Bibliography.

\section{SISTEMAS PRODUTIVOS LOCAIS: ESTRATÉGIAS EMPRESARIAIS PARA 0 DESENVOLVIMENTO}

\section{RESUMO}

O objetivo deste artigo é propor aos sistemas produtivos locais como uma estratégia de industrialização e desenvolvimento. Para cumprir com tal objetivo se apresenta que são teoricamente os sistemas produtivos locais e se expõe o por que são importantes no desenvolvimento. Duas conclusões principais: a primeira é que os sistemas produtivos locais são uma forma de aglomeração empresarial adequada para países como a Colômbia, cujo censo empresarial se compõe quase em sua totalidade por micro e pequenas empresas; e a segunda é que incentivam o desenvolvimento das comunidades, ao pensar o desenvolvimento desde o território mesmo.

\section{PALAVRAS CHAVES}

Desenvolvimento local, corrente produtiva, sistema produtivo local, distritos industriais.

\section{CLASSIFICAÇÃO JEL}

L16, R11, R39.

\section{CONTEÚDO}

Introdução; 1. Sistemas produtivos locais; 2. Economias externas e a configuração dos Sistemas Produtivos Locais; 3. Conclusões; Bibliografia. 


\section{INTRODUCCIÓN}

Los sistemas productivos locales (SPL) pueden definirse como estructuras o modos de organización empresariales orientados a la producción y comercialización de un determinado bien o servicio. Pero estas estructuras encierran una complejidad que es difícil de atrapar en una definición corta, dado el vínculo que estos deben tener con el territorio que los sustenta. Formalmente, el concepto de SPL se deriva de lo que Alfred Marshall llamó en Principles of Ecónomics Distritos Industriales (DI). Los distritos industriales son aglomeraciones de empresas que se dedican en conjunto a la producción y comercialización de un bien o servicio, aglomeración en la cual existen empresas dedicadas a todas las etapas de elaboración del producto: proveedores de materias primas, productores, ensambladores, comerciantes, etc.). De este tipo de aglomeración habló por primera vez el economista inglés Alfred Marshall en su libro titulado Principles of Economics, publicado por primera vez en 1890. En ese libro Marshall comenta una serie de rasgos comunes que encontró en algunas aglomeraciones industriales, como el de cuchillas en Sheffield y el de metal en la ciudad de Birmingham. Los principales rasgos son:

- Un sentido de compañerismo por parte de las empresas, a pesar de estar compitiendo entre ellas.

- Un alto grado de asociatividad que hacía que el Distrito funcionara como una gran empresa, y pudiera generar economías de escala que lo hicieran económicamente competitivo.

- Un mercado laboral en el que sobresalía el aprendizaje empírico, al mejor estilo de los artesanos y sus aprendices.

- Un conocimiento por parte de la comunidad sobre la actividad del distrito, lo que hacía que tuviera una demanda importante.

- La generación de economías externas, como los restaurantes que se ubicaban cerca del servicio, con la finalidad de tener demanda por parte de los trabajadores de este.

Sin embargo, es importante decir que la diferenciación entre DI y SPL no ha sido establecida de manera clara. Por lo tanto, se entiende que un SPL es una aglomeración de empresas que funcionan en mayor o menor medida de manera acorde a lo propuesto por Marshall, como comenta Cividanes (2000). Pero establecer una delimitación tajante es algo que no se ha hecho.

Ahora bien, ¿qué hace tan interesante el estudio de los SPL; qué justifica su estudio? 
Los SPL proponen un cambio de mentalidad frente al que hacer productivo de una sociedad, por varias razones: en primer lugar, es una propuesta distinta al fordismo', filosofía y técnica industrial que cataloga a un proceso productivo como adecuado si este puede producir más y a menor costo un determinado bien; en segundo lugar, es la producción en serie, pero también la separación tajante entre el taller y el diseño, la optimización de la experiencia a través de la repetición para obtener una mayor productividad. Si el fordismo se analiza desde la idea de que el objeto de la producción de un bien solo consiste en producir más del mismo y a bajo costo, resulta ser una forma de producción más adecuada; los inconvenientes vienen al hacer un análisis de las implicaciones sociales que este tiene, lo que en economía se conoce como externalidades ${ }^{2}$ negativas. A continuación se exponen las tres externalidades negativas que genera el modelo fordista:

La primera externalidad es el factor empleo: con la introducción de tecnologías son menos trabajadores de cualquier calificación; luego la innovación tecnológica -si se mantiene constante la cualificación de los obreros- implica desempleo y disminución salarial, que ha conducido a la flexibilización del trabajo como opción en el mercado laboral (posfordismo). Este mismo principio es el que rige las fusiones corporativas, que aumentan su eficiencia a partir de la reducción de personal y la disminución de salarios.

La segunda externalidad es la quiebra que se genera en el mercado de las empresas de menor tamaño, que son incapaces de competir con las economías de escala y los bajos costos de producción de las grandes, lo que deja el liderazgo empresarial solo en manos de los grandes capitales.

La tercera externalidad es el efecto China. China emerge al capitalismo desde una combinación de organización del trabajo muy de cerca al modelo fordista, al proponerse, como país, producir cantidades inconmensurables de diversos artículos, a unos bajos costos, gracias a su tamaño, su régimen político, y las propiedades salariales de su mano de obra, incluso explotada desde opciones flexibles o posfordistas. El efecto China es tan fuerte, que ha impactado el devenir mismo de la civilización actual en múltiples aspectos, como el político, el social, el geográfico; China, y en general Asia generaron en el mundo la deslocalización productiva, y la utilización masiva de mano de obra a precios ínfimos.

\footnotetext{
${ }^{1}$ El fordismo y el taylorismo son en esencia lo mismo. Aquí se utiliza el primer término, dado que es el más conocido.

2 En economía, una externalidad es un efecto indirecto que se da a partir de las relaciones de producción y consumo. Un ejemplo de una externalidad negativa es la contaminación que ocasionan las empresas al producir un determinado bien.
} 
La importancia de los SPL como motores del desarrollo social radica en que son respuesta a estas externalidades, lo que no solo es loable en el ámbito teórico, sino que da cuenta de su efectividad en la economía real, como han demostrado los distritos italianos dedicados a la producción de calzado. Los SPL se constituyen como un factor de desarrollo en el sentido que aspectos como la degradación y destrucción del empleo, el colonialismo industrial y la producción basada exclusivamente en los precios son aspectos negativos (sin tener en cuenta aspectos como la innovación en calidad).

Asimismo los SPL son motores de desarrollo industrial porque funcionan a partir de la elaboración de productos de buena calidad, lo que hace que tengan un lugar particular en el mercado, que no necesariamente les puede quitar un producto más barato; además, esta condición de producir con calidad es una característica que jalona la innovación en el interior de los SPL, porque en la medida que los SPL no tienen una estructura para sobrevivir compitiendo vía costos sino por calidad, estos se ven forzados a innovar y presentar productos cada vez mejores.

Ahora bien, cabe decir que las críticas hechas al modelo fordista en ningún momento son un llamado a acabar con las grandes empresas, sino que son una advertencia sobre las implicaciones negativas que en materia social traen los excesos de esta forma de actividad productiva que no son otras que las consecuencias nocivas propias de los monopolios que están en manos de grandes capitales privados $^{3}$. Ahora bien, da la casualidad de que los monopolios parecieran no poder desprenderse de este modo de producción. No es entonces un inconveniente de las economías de escala, sino de la forma como las grandes empresas, monopólicas en su mayoría, las ejecutan.

El objetivo fundamental de este artículo es hacer una caracterización de los SPL, buscando dar claridad sobre qué es un SPL; qué no es; cuáles son sus ventajas y desventajas; y qué alcances tiene en materia de desarrollo. Para esto, se trabaja a lo largo de este escrito una definición de SPL, la cual busca analizar de forma integral los principales aspectos sobre los cuales se ha reflexionado hasta el momento en lo que a SPL respecta. De manera transversal se argumentará sobre el papel que tienen los SPL en el Desarrollo Local (DL). Por último, se presentan las conclusiones.

\footnotetext{
${ }^{3}$ Basta con un somero análisis histórico para darse cuenta de lo negativa que es materia de generación de bienestar social la actividad monopólica, principalmente la que se ejerce desde los capitales privados. Ahora bien, vale la claridad en cuanto a que este artículo no hace una crítica generalizada al modelo de producción fordista, sino a las implicaciones socialmente negativas cuando este se ejerce en empresas con un poder monopólico.
} 


\section{SISTEMAS PRODUCTIVOS LOCALES}

Los SPL se constituyen espacialmente por empresas que trabajan en torno a la producción y comercialización de uno o varios productos de un mismo sector. Son empresas agrupadas geográficamente, pero cumplen con una serie de condiciones que las hacen ser de mayor complejidad que las aglomeraciones. Las principales características se pueden resumir así:

- Las empresas pertenecientes a la aglomeración son en su amplia mayoría micro, pequeñas y medianas empresas (Mipymes).

- Existen empresas que operan en los eslabones de la cadena productiva y comercializadora de los productos que son centrales en la aglomeración.

- No hay una empresa que tenga un poder de mercado lo suficientemente grande como para subyugar la actividad productiva y comercial de las otras empresas de la aglomeración.

- Hay una dinámica de asociatividad entre y desde las empresas, que potencia la competitividad, la productividad y la innovación.

- El SPL puede trascender la aglomeración y ser capaz de nuclear algunas en diferentes espacios

Además de estas características fundamentales, también hay elementos que se identifican en el proceso de construcción de los SPL, que pueden hacerse extensivos a todas las aglomeraciones productivas. El gráfico 1 los ilustra:

Gráfico 1. Composición de una aglomeración productiva ${ }^{4}$

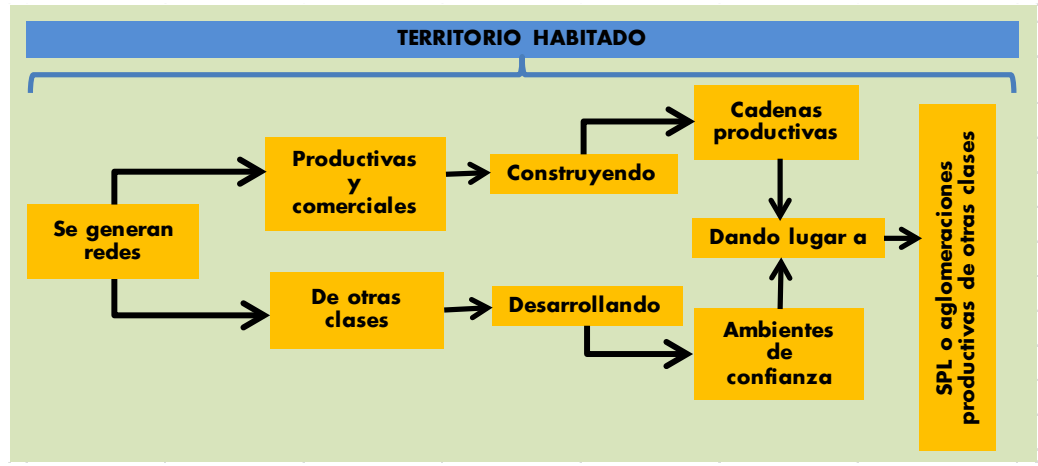

Fuente: elaboración propia

${ }^{4}$ En el gráfico 1, cuando se plantean "otras aglomeraciones productivas", se hace referencia a aquellas que en mayor o menor medida se acercan a un SPL. Por ejemplo, en España se habla de Sistemas Locales de Empresas (SLE), y se reconoce que este término es sinónimo de SPL. Lo que se tiene entonces son aglomeraciones productivas, y en mayor o menor medida tienden a ser SPL, y eventualmente Distritos Industriales, como se verá más adelante. 
El análisis de estas especificidades es esencial para comprender el funcionamiento y las implicaciones económicas de los SPL, que no pueden ser el objetivo, sino el medio para que un territorio confluya hacia mejores condiciones de vida.

De esta manera, las redes (networks) se constituyen en una parte esencial del funcionamiento de los SPL; no son otra cosa que relaciones de cooperación que se generan a partir de dos o más agentes. Se puede afirmar que las redes tienen distintas funciones, nacen a partir de diversos objetivos y toman diferentes formas y tamaños, como indica Castells (2006), quien también muestra que la identificación y entendimiento de una red depende del análisis particular de la misma. Es decir, donde hay cooperación de forma repetida existe una red, pero es en el análisis particular donde se entiende la dinámica de cada una. Las redes son las unidades más pequeñas de un SPL; es decir, para que exista un SPL tiene que existir por lo menos una red identificable, pero es claro que en los SPL se generan redes de producción, de colaboración y de soporte.

Las dinámicas productivas a través de redes requieren entender los limitantes de concebir la competitividad como un asunto empresarial, e involucran componentes integradores, como la mesoeconomía, los consensos sociales o la política económica, que contribuyen a un entendimiento del fenómeno de la producción, la innovación y la competitividad como asuntos complejos que requieren de la integralidad de análisis, saberes y acciones (Rendón, 2012, p. 87).

Otro componente esencial son las cadenas productivas; para que exista un SPL debe estar presente una intención coordinada de producción y comercialización, y esto implica un trabajo conjunto entre empresas, el cual va desde los primeros tratamientos de una materia prima, hasta la venta del producto final. Cabe aclarar que la existencia de una cadena productiva no implica la existencia de un SPL, pero este no es concebible si dentro de él no se identifican cadenas productivas. Si se analiza a fondo, se encuentra que las cadenas productivas se componen de redes, orientadas hacia la producción y puesta en el mercado de un producto (Forero, 2013).

Sin lugar a dudas un SPL es identificable en un territorio, es decir, el SPL se ubica en el espacio pero aporta a la construcción del territorio. El término implica una serie de condiciones producidas por la diaria convivencia de quienes lo habitan, es decir, el territorio como espacio de construcción social, el lugar de intercambios y relaciones humanas, materiales y con la naturaleza. Se puede afirmar que el territorio es un espacio de potencialidades que pueden ser aprovechadas de distintas formas por parte de quienes integran un SPL; como afirman Álvarez y Rendón (2010, p. 39) "los territorios no son un factor de competitividad en sí mismos, más bien, 
cuentan con potencialidades que pueden o no ser aprovechadas, según las decisiones políticas que se adopten".

Ahora bien, el territorio no puede ser visto de ningún modo como un factor independiente que suma o resta según se tenga en cuenta o no5 . Como se advirtió anteriormente, el territorio es una configuración compleja de carácter geográfico, histórico, político, entre otros aspectos; por ende, la producción en el territorio es un producto de este y, a la vez, un determinante de cómo se configurará el territorio en el presente y hacia el futuro. Es necesario tener siempre claro que "Enfrentarse al planteamiento de la localidad como alternativa de desarrollo territorial significa repensar la función que el territorio cumple dentro de la dinámica de la producción y, junto a ella, las cadenas de relaciones que se generan en el interior y para el exterior de las localidades" (Álvarez y Rendón, 2010, p. 54). Son entonces los SPL un elemento del desarrollo local, y a la vez un producto de las dimensiones no necesariamente productivas de este.

\section{ECONOMÍAS EXTERNAS Y LA CONFIGURACIÓN DE LOS SPL}

Junto a los conceptos de rendimientos crecientes y competencia imperfecta aparece el de economías externas, para ayudar a conceptualizar el fenómeno conocido como aglomeraciones productivas en tanto motores de crecimiento y desarrollo, tal como advirtiera Marshall $(1920)^{6}$, componentes esenciales del desarrollo endógeno. Las economías externas son dispositivos fundamentales de los SPL, son transversales a su funcionamiento y, a la vez, estructuran su composición, en lo que a innovación, productividad, eficiencia y mejoramiento de la calidad de vida se refiere. Las economías externas como plantean Hernández y Soler (2008, pp. 84 85):

Se generan gracias a la «red» de relaciones inter empresariales que se establecen en el seno de estas aglomeraciones industriales permitiendo una «especialización en

5 Desde las concepciones básicas de la economía se habla de la tierra, el trabajo y el capital como los componentes fundamentales de la producción, y se traduce esta creencia en una función de la forma $\mathrm{P}=\mathrm{f}(\mathrm{T}, \mathrm{Tr}, \mathrm{C})$ : $\mathrm{P}=$ Producción, $\mathrm{T}=$ Tierra, $\mathrm{Tr}=$ Trabajo, $\mathrm{C}=$ Capital. La concepción del territorio en el Desarrollo Local (DL) y por ende en el estudio de los SPL es más compleja, porque no es un elemento que se adiciona o se retira a voluntad, ni siquiera en la concepción teórica, es un elemento indivisible de la producción y la actividad de la aglomeración. Concebirlo de la forma convencional anterior es un sinsentido equivalente a cuando se considera que el capital y el trabajo son perfectamente sustituibles.

6 Marshall (1920) se interesó por la forma como operaban estas estructuras en Inglaterra, y extendió el análisis a aspectos como las relaciones económicas que se generan alrededor de las aglomeraciones, como los restaurantes que se ubican en la zona para nutrir la demanda con los trabajadores de las industrias establecidas, entre otros; a este tipo de relaciones colaterales se les conoce actualmente como economías externas, y son propias de casi todas las aglomeraciones. Becattini, Costa y Trullén (2002, pp. 29-39), mencionan tres anomalías marshallianas: La primera, "el hombre concebido no como un muñeco que maximiza una función de utilidad [... sino como una entidad variable, plástica, que produce el ambiente social y que es, a su vez, producida según leyes complejas y desconocidas, también por poco investigadas, que se enlazan con la simple mecánica de los intercambios"; la segunda anomalía es, el Distrito Industrial, y la tercera es "el proyecto de aplicar dichas curvas [de comercio internacional] a las relaciones entre agrupaciones sociales compactas". 
fases» de la producción. Además de poder contar con un mercado laboral denso y de trabajadores cualificados, con una atmósfera que propicia la innovación y el carácter emprendedor del empresario, y una importante fluidez de información de todo tipo entre las propias empresas. Un entorno, en definitiva, que combina competencia y colaboración inter empresarial.

Así, se hace necesario resaltar el legado de Marshall (1920), y comprender las economías externas como el resultado del aprendizaje colectivo que se genera a partir de la interacción entre las empresas de una determinada aglomeración, lo que lleva a dinámicas de innovación, estabilidad productiva y comercial, y generación de bienestar colectivo. En el cuadro 1, se presentan distintas concepciones teóricas al respecto. Es importante resaltar que no se deben confundir las economías externas con las externalidades, dado que las segundas se refieren a los resultados de la producción que no se expresan en el precio; mientras que las primeras recogen los efectos de la producción y, adicionalmente, de las relaciones sociales de diferente clase que se dan en una aglomeración y, por lo tanto, en un territorio definido.

Cuadro 1. Concepciones sobre las economías externas

\begin{tabular}{|l|l|}
\hline \multicolumn{1}{|c|}{ Posición } & \multicolumn{1}{c|}{ Concepción } \\
\hline $\begin{array}{l}\text { Marshall } \\
\text { (1920) }\end{array}$ & $\begin{array}{l}\text { Categoría atada a los distritos industriales y a los spillovers tecnológicos } \\
\text { y de conocimiento. Se refiere a la difusión espontánea de conocimiento } \\
\text { generada por la especialización en el mercado de trabajo y en el mercado } \\
\text { de bienes intermedios y de servicios a las empresas, que garantizan una } \\
\text { espiral de innovación para la industria. }\end{array}$ \\
\hline $\begin{array}{l}\text { Hoover } \\
(1937)\end{array}$ & $\begin{array}{l}\text { Realiza una distinción entre economías de localización, esto es a decisiones } \\
\text { racionales de óptimos económicos de la empresa frente a la localización } \\
\text { de otras empresas de la misma industria y economías de urbanización que } \\
\text { trascienden la industria o la actividad económica y se refieren igualmente } \\
\text { a la localización. }\end{array}$ \\
\hline $\begin{array}{l}\text { Scitovsky } \\
(1954)\end{array}$ & $\begin{array}{l}\text { Se propone una distinción entre economías externas pecuniarias y } \\
\text { tecnológicas. Las primeras se refieren a los menores costos que en los } \\
\text { distritos industriales se tiene de mano de obra especializada, así como } \\
\text { de las materias primas y los servicios a la producción. Las tecnológicas } \\
\text { tienen que ver con la difusión del conocimiento y el progreso técnico. }\end{array}$ \\
\hline Jacobs (1969) & $\begin{array}{l}\text { Se plantean los spillovers tecnológicos y de conocimiento no desde la } \\
\text { especialización, al estilo Marshall, sino desde la diversificación productiva. } \\
\text { Las externalidades tipo Jacobs hacen referencia a economías externas } \\
\text { producto de acciones económicas intersectoriales. }\end{array}$ \\
\hline
\end{tabular}




\begin{tabular}{|l|l|}
\hline \multicolumn{1}{|c|}{ Posición } & \multicolumn{1}{c|}{ Concepción } \\
\hline $\begin{array}{l}\text { Marshall } \\
\text { (1920), Arrow } \\
\text { (1962) y } \\
\text { Romer (1986) }\end{array}$ & $\begin{array}{l}\text { Se conoce como externalidades tipo MAR }{ }^{7} \text {, a partir de estos trabajos } \\
\text { empresas del mismo sector, e incluso intrasectoriales. }\end{array}$ \\
\hline $\begin{array}{l}\text { Glaeser et al. } \\
(1992)\end{array}$ & $\begin{array}{l}\text { Este asume que las externalidades tipo MAR se benefician de la escasa } \\
\text { competencia, a diferencia de las de Jacobs donde la competencia es fun- } \\
\text { damental para potenciarlas. Propone una nueva categoría: Externalidades } \\
\text { tipo Porter, donde se asume la competencia en las externalidades tipo } \\
\text { MAR. } \\
\text { Se clasifica las economías externas de localización en economías externas } \\
\text { estáticas intrasectoriales, y en economías externas de urbanización como } \\
\text { dinámicas e intersectoriales. }\end{array}$ \\
\hline $\begin{array}{l}\text { Oughton } \\
\text { y Whittam } \\
(1997)\end{array}$ & $\begin{array}{l}\text { Se propone una categoría adicional a las expuestas por Scitovsky (1954), } \\
\text { las economías externas colectivas. Estas se dan cuando las empresas coo } \\
\text { peran entre sí para obtener economías de escala en producción, compras } \\
\text { capacitación o difusión de conocimiento productivo o de mercados. Son } \\
\text { externas a la empresa pero internas al grupo de empresas que participan } \\
\text { en el proceso colectivo de intercambio y cooperación. }\end{array}$ \\
\hline $\begin{array}{l}\text { Bellandi } \\
\text { (2002) }\end{array}$ & $\begin{array}{l}\text { Clasifica las economías externas en economía de especialización, apren- } \\
\text { dizaje, y creatividad: Las primeras tienen las economías de escala que se } \\
\text { generan en el Distrito. Las segundas se dan a partir de los aprendizajes } \\
\text { que se producen en el distrito por la interacción entre los agentes que } \\
\text { lo componen. Finalmente, las economías externas de creatividad son el } \\
\text { resultado del intercambio de información dentro del Distrito, el cual lleva } \\
\text { a dinámicas de innovación tanto de proceso como de producto. }\end{array}$ \\
\hline
\end{tabular}

Fuente: elaboración propia, con base en rendón (2009, pp. 100-101).

Las economías externas son particularmente importantes en aglomeraciones y en los SPL donde la innovación es un objetivo que busca convertirse en el soporte de más y mejores formas de producción y comercialización. Según Oughton y Whittam (1997), la planificación permite explotar mejor las economías externas y, a su vez, estas posibilitan construir procesos innovadores. La planificación resulta inherente a la supervivencia misma de las empresas; contrario a lo que se dice desde la teoría neoclásica, el empresario no opera de manera racional y con información completa; la planificación es importante precisamente para enfrentar la incertidumbre del entorno, y su objetivo es permitir de manera coordinada el uso de la innovación, los aprendizajes y las tecnologías; se da obviamente en y desde la empresa pero

\footnotetext{
7 Aunque se podría decir que las externalidades tipo MAR (Marshall, Arrow, Romer) son comparables con las economías externas.
} 
también en la configuración propia del territorio, en la búsqueda de instituciones propicias que fomenten la gobernabilidad y la gobernanza en la localidad.

No se debe olvidar que el entorno está enmarcado en un territorio, y este, a su vez, está configurado por una serie de factores que en él operan; como son las instituciones, las empresas, los recursos naturales, las redes, entre otros. Esto hace que el territorio termine por influenciar los procesos de I+D+I que realicen las empresas. De esta manera no son las empresas solas las que compiten, lo hacen con las sinergias propias de los SPL y del territorio, es decir, aprovechan las economías externas y potencian sus propios desarrollos (Rendón, 2009, 100).

Las economías externas son entonces inherentes al territorio, y muestran que la economía es un entramado complejo de relaciones que van desde las propias relaciones de producción y pasan por la política, la cultura y el medio ambiente. Como Alburquerque (2005, p. 7) afirma, "el análisis del desarrollo económico en la práctica, involucra o exige un enfoque integrador de todos estos aspectos relativos al desarrollo socioeconómico, el desarrollo institucional, el desarrollo humano y el desarrollo sostenible".

Marshall (1920) encontró que no solo había SPL -aunque él no utilizó este término- sino que aparte de lo mencionado, identificó la existencia de una cultura alrededor del SPL, una demanda que, más allá del factor precio, consumía los productos de dicha aglomeración por un aspecto cultural; a estos SPL que tienen una demanda existente por un aspecto que trasciende el factor precios, y que genera economías externas, se le conoce como Distrito Industrial (DI), y es el estado óptimo de un SPL; es decir, es hacia lo que un DI debe evolucionar. Si bien el límite donde una aglomeración pasa a ser un SPL, o donde un SPL pasa a ser un DI no es claro, hay algunas propuestas para la caracterización de estos estados de aglomeración productiva. En este trabajo se destaca Climent (2008, p. 43), quien a partir de Garofoli (1992), expone la tipología que el académico italiano propone:

Área de especialización productiva: área local donde se concentra un cierto número de pequeñas y medianas empresas dedicadas a la misma actividad, sin que lleguen a mantener relaciones entre ella más allá de la competencia por los mismos mercado.

Sistema productivo local: empiezan a tejerse algunas relaciones de mercado y de cooperación entre las empresas dedicadas a la mima actividad, pero la división técnica del trabajo de carácter vertical es aún débil.

Área-sistema: es un auténtico distrito industrial con una profunda división del trabajo, tanto vertical como horizontal, y relaciones de cooperación entre las empresas y entre estas y las instituciones locales ${ }^{8}$.

8 Es posible pensar que de acuerdo con el grado de organización se tiene una secuencia evolutiva aglomeración $\rightarrow \mathrm{SPL} \rightarrow \mathrm{DI}$; si bien algunos estudios no utilizan el término SPL, sino otros como Sistema Local de Empresas (SLE), autores como el mismo Climent (2008, p. 43) advierten que "Los términos sistema productivo local y sistema local de empresas pueden considerarse sinónimos", esto es algo que permite 
Climent (2008, p. 43) agrega que "Es difícil establecer límites precisos entre los tres tipos, aunque la diferencia entre el primero y el último es clara. Por otra parte, puede darse una trayectoria evolutiva entre los tres tipos, pero no necesariamente".

Desde la existencia misma de los distritos industriales se intuye la capacidad de las localidades para generar el desarrollo, gracias a la flexibilidad y movilidad de los factores de producción; pero esto no debe confundirse con el deterioro integral de las condiciones de trabajo que con tanto entusiasmo se justifica, en especial en las economías subdesarrolladas, en pro de una supuesta eficiencia y competitividad; flexibilidad para asumir nuevas producciones, movilidad para atender requerimientos disímiles, para soportar entornos innovadores, para trascender los propios espacios de la producción, la comercialización y el consumo.

Se trata, no obstante, de la capacidad de los SPL para convertirse en una nueva y distinta dimensión de la innovación, donde se generan economías externas, entornos innovadores capaces de apalancar nuevos emprendimientos y eslabonamientos hacia atrás y hacia adelante en los procesos productivos, de tal forma que en el proceso mismo se contribuye a mejores estadios de crecimiento productivo y desarrollo.

Ahora, se debe reconocer que las Mipymes se han constituido en parte táctica y estratégica del actual modelo dominante, desde la gran empresa, para adecuarse a la flexibilidad de los procesos productivos, para tener en la descentralización y deslocalización factores importantes para la reducción de costos y convertir a la pequeña empresa en proveedora de servicios, integrada, sin mayores responsabilidades contractuales, a los procesos productivos y por fuera de la organización y la comercialización (Rendón, 2012, p. 87). Precisamente los SPL se soportan en las Mipymes para articularlas en torno a propósitos que se territorialicen (convirtiéndose en DI) y no al servicio de una empresa grande, probablemente transnacional, e incluso articulada a clusters al servicio de estas.

En definitiva es característica fundamental de los SPL la de adaptarse a las cambiantes circunstancias de los entornos o a la necesidad de establecer la innovación y el cambio permanente como estrategia de acceso a los mercados, sean estos locales, nacionales o globales y generar así entornos innovadores, competitivos, especializados y flexibles que logren capturar sinergias propias combinadas con rápidas acciones en los mercados globales para adaptarse a las cambiantes circunstancias de las economías internacionales (Rendón, 2012, p. 86).

Se tiene, entonces, que cada aglomeración productiva, y por ende cada SPL,

homologar los análisis y darles un cauce común. Por otro lado, es importante aclarar que un SPL no tiene solo la opción de migrar hacia DI, sino que también puede hacerlo hacia un Clúster, que es un modo de organización industrial propuesto desde Norte América, especialmente por Porter, (1990). 
es única, y su identidad está determinada por los factores históricos, geográficos, institucionales, de diversa índole, que a lo largo de su constitución y funcionamiento han influido en la configuración de las relaciones y consecuentemente de las redes que la componen.

Ahora bien, después de Marshall (1920) otros pensadores retoman sus propuestas. Esto sucedió particularmente en el sur de Europa; Becattini (2002) es el más reconocido de ellos, quien se concentró en estudiar los SPL europeos, y en especial los italianos, donde observó la existencia de SPL alrededor del calzado. Becattini (2002) encontró que estos distritos de calzado resultaban interesantes, aparte de las condiciones ya mencionadas, por varias razones que a continuación se enumeran:

- Son grandes generadores de empleos directos, e indirectos, tanto por la actividad central del distrito, como por las economías externas adyacentes a este.

- Generan dinámicas de innovación, a pesar de estar compuestos por Mipymes.

- El estatus competitivo en los mercados se basa en la calidad, no en la producción de grandes volúmenes a bajo costo.

- El conocimiento se transmite de generación en generación; el saber hacer del calzado pasa de los trabajadores a los aprendices que se vinculan al distrito.

- Existe una cultura de "comprar en el distrito" lo que dificulta la entrada de nuevos competidores, por más bajos que fueran los precios.

- Si se requieren grandes volúmenes de producción en el distrito confluyen las empresas que con base en los conocimientos compartidos (bajo los principios de asociatividad, cooperación y solidaridad) son capaces de estandarizarla a partir de unidades de producción diferentes.

Estas características de un SPL son las que hacen de esta forma de organización productiva deseable en pro de generar bienestar social, dado que son motor de empleo y activación económica visible y palpable para los integrantes de una localidad; por esto, son un componente fundamental del desarrollo local. El desarrollo implica abordar varias dimensiones: políticas, sociales, productivas, institucionales etc. que se entrelazan entre sí. Los SPL se constituyen en la dimensión productiva del desarrollo local, lo que permite la materialización de las relaciones sociales de producción.

Vale la pena resaltar algunas definiciones propuestas por estudiosos de los SPL; en el cuadro 2 se presentan algunas: 
Cuadro 2. Definiciones de SPL

\begin{tabular}{|l|l|}
\hline $\begin{array}{l}\text { Cividanes } \\
(2000, \text { p. 1) }\end{array}$ & $\begin{array}{l}\text { "Término acuñado para referirse de manera genérica a todos aquellos casos } \\
\text { de concentración espacial de la actividad industrial cuyo estudio se aborda } \\
\text { aplicando como marco teórico, en mayor o menor medida, el concepto } \\
\text { socioeconómico de distrito industrial". }\end{array}$ \\
\hline $\begin{array}{l}\text { "Se trata de un área local con una marcada especialización industrial a base } \\
\text { de pequeñas y medianas empresas (PYME a partir de ahora), entre las que } \\
\text { existe una fuerte división técnica del trabajo, en el sentido horizontal (espe- } \\
\text { cialización de gama) y vertical (especialización de fase). Entre las empresas } \\
\text { p. 40) }\end{array}$ & $\begin{array}{l}\text { del distrito hay relaciones de competencia y de cooperación, así como una } \\
\text { atmósfera industrial, término que hace referencia tanto a conocimientos } \\
\text { teóricos, productivos y de gestión, como a un sistema de valores compartido }\end{array}$ \\
\hline $\begin{array}{l}\text { yecattini } \\
\text { y Musoti } \\
(2008,\end{array}$ & $\begin{array}{l}\text { "Los distritos industriales pueden describirse como un tipo de proceso en } \\
\text { forma de espiral que envuelve por completo a una comunidad local y a su } \\
\text { p. 75) } \\
\text { exterior tiene sobre el capital, en forma de nuevas empresas o la disolución } \\
\text { de compañías locales previamente existentes". }\end{array}$ \\
\hline $\begin{array}{l}\text { Vázquez } \\
(2000, \\
\text { p. 25). }\end{array}$ & $\begin{array}{l}\text { "Un sistema productivo local es más que una red de empresas, ya que está } \\
\text { integrado también por una red de actores sociales compuesta por una serie } \\
\text { de relaciones económicas, sociales, políticas y legales [... De este modo, } \\
\text { el sistema productivo local es un entorno (milieu) que integra y domina un } \\
\text { conocimiento, unas reglas, unas normas y valores, y un sistema de relaciones". }\end{array}$ \\
\hline $\begin{array}{l}\text { Flórez y } \\
\text { parroso }\end{array}$ & $\begin{array}{l}\text { Un SPL se caracteriza porque "la organización de la producción se realiza } \\
\text { mediante una red de pequeñas y medianas empresas, siendo el sistema de } \\
\text { relaciones que se establecen entre ellas lo que hace que la organización de } \\
\text { la producción sea eficiente y competitiva en ese territorio". }\end{array}$ \\
\hline
\end{tabular}

Fuente: elaboración propia.

Algunas cosas a resaltar sobre el cuadro 2: la definición de Vázquez (2006) es una cita de Maillat (1996), que además recoge los postulados de Becattini (2002) sobre los SPL, También se puede ver que Cividanes (2000) es el único que habla puntualmente de los SPL, aunque concluye que estos son estados imperfectos de un DI. De resto, todas las definiciones hablan puntualmente de los DI.

Es menester entonces centrar la discusión en el tema de los DI. El primer DI del que se tiene registro es obviamente el analizado por Marshall (1920), quien enfoca la atención en dos industrias: la cuchillería de Sheffield y la del metal de Birmingham (Becattini, 2002). Extendiéndose el análisis al resto de Europa, se encuentran los distritos de calzado en Italia, también en España, y en Francia; pero no solo de 
calzado, sino que estas aglomeraciones son visibles en estos países en las industrias del vestido, los muebles -caso España-, los textiles y las confecciones, entre otros.

En Latinoamérica llaman particularmente la atención los SPL de calzado, que bajo un esfuerzo coordinado entre Estado y empresarios ha logrado posicionarse en el mercado internacional; se resalta el caso de Brasil, que exporta calzado de alta gama a los Estados Unidos. También vienen dándose casos importantes de organización productiva bajo esquemas asociativos en Perú, Chile, Argentina y México. Esto resulta interesante por cuanto dan cuenta de un hecho altamente relevante; que los SPL son una alternativa a la organización industrial implementada por el fordismo y el taylorismo, que traen beneficios palpables para las sociedades, y son motores de desarrollo local por cuanto su papel como generadores de empleo, estabilidad económica y dinamización industrial es directo. En Colombia apenas se están realizando análisis de las aglomeraciones (donde se habla indistintamente de clúster) como formas de tratar de conducir las decisiones públicas (locales, como en los casos de Bogotá y Medellín fundamentalmente) y reconfigurar con ello la política industrial que se ha basado en normas y acciones dispersas que difícilmente constituyen un cuerpo sólido para la acción; sin embargo, aún se está lejos de pensar en torno a DI.

Queda un último elemento por mencionar, pero no menos importante; el papel del Estado en la configuración y sostenimiento de los SPL. ¿Son los DI aglomeraciones de generación espontánea, ${ }^{9}$ o alguien los configura? Becattini (2002, p. 16) responde que las dos cosas son posibles e instaura los conceptos de distritos semiautomáticos y distritos construidos. Sobre los primeros afirma Becattini $(2002$, p. 16) que se dan a partir de la creciente especialización en una aglomeración ya en marcha, y agrega que "se trata de un largo y complicado proceso en el que un continuo flujo de innovaciones socioeconómicas se mezcla con una sucesión de consolidaciones de las más eficientes innovaciones en específicas instituciones". Por otro lado, los distritos construidos, indica el autor, tienen cierto grado de intervención consiente para su creación.

Lo que muestra actualmente la evidencia es que más allá de cómo se da la generación de las aglomeraciones, estas muy difícilmente llegan a ser DI sin una intervención por parte del Estado; en el mejor de los casos pueden llegar por sí solas a SPL, pero los cambios actuales, la liberalización económica, el dumping, la

Hablar de generación espontánea es diferente a decir "salieron de la nada". La espontaneidad indica que algo surge a partir de condiciones complejas. Es decir que el surgimiento de una aglomeración se denomina "espontáneo" cuando se da a partir de factores culturales, demográficos, socioeconómicos, políticos y geográficos que de manera única confluyen en un momento específico y dan lugar a la aglomeración, siendo muy difícil en muchos casos la sola identificación de dichos factores. 
complejidad de la cultura y el veloz progreso tecnológico han hecho que sin un ente que estructure, oriente y fortalezca a las aglomeraciones, estas están destinadas al fracaso. Es decir, la política industrial, tanto desde los entes nacionales como locales, y de las instituciones que la soportan, se constituye en elemento fundamental para consolidar procesos de aglomeración y, con ellos, formas cada vez de mayor cualificación. Se dice "evidencia" porque al igual que los casos mencionados, es un hecho la necesidad de esta intervención: lo fue para los distritos ingleses, para los italianos, y fue lo que permitió puntualmente a los SPL de calzado brasileros alcanzar el estado de DI y obtener resultados virtuosos. De tal modo que la estructuración de los SPL justifica el fortalecimiento institucional, los acuerdos sectoriales, la participación empresarial y obviamente la intervención estatal decidida a través de políticas industriales.

\section{CONCLUSIONES}

El desarrollo como proceso de mejoramiento de las condiciones de vida de las personas no se puede concebir sin tener en cuenta la dimensión de lo local. Es en el día a día de las personas, en su contexto inmediato, donde transcurren sus vidas, donde se produce, se comercializa, se consume y se tejen las relaciones que procuran los posicionamientos políticos, la consolidación de las culturas; y es ahí donde se debe intervenir para que las personas efectivamente puedan mejorar la calidad de vida, a partir de sus propias definiciones de futuro, al diseño de un buen vivir.

No quiere decir esto que la planeación y ejecución de proyectos de desarrollo macro carezca de sentido, pero sí es innegable que el diseño de dichas políticas está incompleto cuando se hace -como pasa en la mayoría de casos- desde la idea de un espacio o incluso de individuo plano y etéreo. Es decir, no se puede planificar el desarrollo sobre un modelo de sociedad e individuo que no existe. Este no es propiamente el individuo aislado que concurre al mercado en busca de su propio bienestar; se trata, eso sí, de entender que las sociedades se construyen a partir de seres sociales que buscan, como individuos, familias y sociedad, una mejor condición de bien-estar.

Los SPL como aglomeraciones productoras y comercializadoras que funcionan a partir de Mipymes tienen tres componentes fundamentales: las redes, las cadenas productivas y el territorio. Estos componentes son un esbozo conceptual para orientar su análisis, estructuración y fortalecimiento. Sin embargo, estudiar los SPL requiere plena conciencia sobre el hecho de que cada aglomeración requiere un análisis particular. No se puede planificar el trabajo sobre SPL y asumir que todos son iguales, el análisis resultaría excesivamente obvio o incorrecto, y la ejecución hecha a partir del mismo sería probablemente errada. 
Por otro lado, el funcionamiento de los SPL a partir de redes y cadenas productivas compuestas Mipymes tiene una serie de implicaciones de carácter socioeconómico que bien vale la pena resaltar: la primera es el papel de este tipo de aglomeraciones en la generación de empleo, el cual se mantiene de forma sostenida gracias a que estas aglomeraciones son estructuras sólidas que, como sucede en escenarios concretos referenciados con el calzado, los textiles o la moda, generan una afiliación de su demanda, por lo menos, en el ámbito local, y la proyectan a espacios nacionales e internacionales.

La segunda es que en estas aglomeraciones coexiste la competencia y la cooperación, algo impensado en el modelo neoclásico. Por otro lado, la creación de un ambiente en el que la asociatividad y la competencia no son condiciones excluyentes permite que se den dinámicas de innovación, así como la generación de entornos virtuosos e innovadores.

Los SPL son entonces una alternativa posible, dado que no solo constituyen un modo de organización empresarial que emerge a la estructura productiva existente sino que responde a una serie de problemáticas que tienen asidero fundamentalmente en la debilidad industrial propia del mundo moderno en los países subdesarrollados.

Es la producción a partir de aglomeraciones de Mipymes una alternativa que ha dado resultados interesantes, que, por demás, posee una base conceptual y un soporte académico, que ofrece soluciones alternativas a fórmulas mágicas como la introducción de grandes capitales sin importar su destino o finalidad, la exención tributaria a empresas poco intensivas en mano de obra e innovación, o los incentivos destinados exclusivamente a los grandes exportadores; medidas que no han arrojado los resultados esperados en materia de progreso económico y bienestar social, argumentos que se utilizaron para implementarlas.

Para finalizar, vale la pena reflexionar sobre algunos temas que resulta pertinente resaltar:

El primer aspecto es la concepción económica de los SPL. El análisis económico está soportado sobre una preconcepción filosófica del ser humano, la sociedad y el deber ser de esta. La idea de los SPL resulta absurda a la luz de la teoría económica ortodoxa, y es inentendible desde este modelo de análisis. No en vano Becattini (2002) comenta que los DI son la segunda anomalía marshalliana, porque no encajan en el razonamiento de la economía ortodoxa (ni la de la época de Marshall, que curiosamente él ayudó a crear, ni en la de ahora). Por eso hay una invitación clara a pensar la humanidad, la sociedad y el deber ser de la misma desde otras lógicas, 
desde otra filosofía, bajo otros parámetros, y esto es importante por cuanto construir un soporte teórico sólido, que permita una modelización adecuada para los SPL y para el desarrollo local, se convierte en un reto, en escenarios donde lo local y lo global se enlazan para posibilitar nuevas formas de progreso.

Lo segundo es el factor asociativo. Este es un elemento fundamental en los SPL porque es esa conciencia de cooperación mutua, de saber que se pertenece a algo más grande por parte de los empresarios lo que permite que este tipo de agrupación industrial funcione, dado que el actuar de cada empresario influye en el bienestar de los otros, lo que los lleva a operar de manera adecuada y a velar por que los demás lo hagan; y esto incluso aplica a temas distintos a la producción, trasciende a temas como participación política, planificación territorial, educación, entre otras. La apuesta por modelos asociativos, de cooperación productiva no debería ser solo obra de las Mipymes; la política pública deberá proporcionar los elementos para que la gran empresa sea partícipe de estos procesos.

En tercer lugar, se debe hacer un llamado, y en especial en países como Colombia, a que se produzca información útil para poder hacer análisis de SPL. En general conforme se replantea la reflexión en torno al desarrollo local y se piensa la modelización de este, se deberán diseñar métodos de medición y estimación estadística alternativos. Habrá que establecer otras lógicas para entender y medir las dinámicas de concentración espacial y relacionarlas con otros parámetros, pero para que estos ejercicios de medición y estimación sean pertinentes en el diseño, ejecución de política pública y planeación industrial es necesario que existan los datos que permitan hacer esto. Es decir, se trata de la discusión sobre la unidad de análisis; sea esta el SPL o el DI, la información estadística debería ser consecuente con ello. Hoy lo es frente al sector, aspecto que impide un análisis concreto y exige que los estudios deban partir de la recolección primaria de información, de los estudios de caso como razón de entender las dinámicas sociales en la empresa o el conjunto de empresas en el territorio.

\section{BIBLIOGRAFÍA}

Alburquerque, Francisco (2005). Reflexiones sobre el desarrollo económico en la práctica: Desarrollo y territorio. Universidad Complutense de Madrid, Madrid.

Arrow, Kenet (1962). The economic implications of learning by doing. En: Review of Economic Studies, N. ${ }^{\circ} 29$, pp. 155-173.

Álvarez, Rubén y Rendón, Jaime (2010). El territorio como factor de desarrollo. En: Semestre Económico, Vol. 13, N. 27, Universidad de Medellín, Medellín, pp. 39-62. 
Sistemas productivos locales: estrategias empresariales para el desarrollo

Becattini, Giacomo (2002). Del distrito industrial marshalliano a la "teoría del distrito" contemporánea. Una breve reconstrucción crítica. En: Investigaciones Regionales, N. 1, pp. 9-32.

Becattini, Giacomo, Costa, María y Trullén, Joan (2002).Desarrollo local: teorías y estrategias. Editorial Civitas Ediciones, Madrid, 339 pp.

Becattini, Giacomo y Musotti, Francesco (2008). Los problemas de medición del "efecto distrito". En: Mediterráneo Económico, N. ${ }^{\circ}$ 13, pp. 55-82.

Bellandi, Marco (2002). Italian industrial districts: and industrial economics interpretations. En: European Planning Studies, N. ${ }^{\circ}$, pp. 425-437.

Castells, Manuel (2006). La sociedad red. Alianza Editorial, Madrid, 557 pp.

Cividanes, José (2000). El territorio como variable económica: El concepto de sistema productivo local. Universidad de Alicante, Dpto. Análisis Económico Aplicado, España, 13 pp.

Climent, Eugenio (2008). Los nuevos retos de los sistemas locales de empresas: entre la tradición y la innovación. En: Martínez, Antonio., Rodríguez, Fermín y Prieto, Antonio (coords). Los sistemas locales de empresas y el desarrollo territorial: evolución y perspectivas actuales en un contexto globalizado, Editorial Club Universitario, Alicante, España, 445 pp.

Flórez, David y Barroso, María de la O. (2010). Materiales tema 3: Sistemas productivos locales. Universidad Internacional de Andalucía, OpenCourseWare Web.

Forero, David (2013). El sector del calzado en el barrio El Restrepo, Bogotá. Un análisis de caso a la luz de los sistemas productivos locales. Universidad de La Salle, Programa de Economía, Tesis de grado, Bogotá, Colombia, 81 pp.

Garofoli, Gioacchino (1992). Endogenous development and southern Europe. USA: 240 pp.

Glaeser, Edward; Kallal, Hedy; Scheinkman, J y Shleifer, Andrei (1991). Growth in cities. National Bureau of Economic Research, N. ${ }^{\circ} 3787,38$ pp.

Hernández, Francesc y Soler, Vicent (2008). Medición del "efecto distrito": Una aproximación no paramétrica. En: Mediterráneo Económico, N. ${ }^{\circ}$ 13, pp. 83-96.

Hoover, E. M (1937). Locations, Theory and the Shoe and Leather Industries. Cambridge: Harvard university press, $323 \mathrm{pp}$.

Marshall, Alfred (1890). The Principles of Economics. London: MacMillan, 627 pp.

Marshall, Alfred (1920). Industry and trade. England. 681 pp.

Maillat, Denis (1996). From the industrial district to the innovative milieu: Contribution to an analysis of territorialized productive organizations. En: Recherches Economiques de Louvain, Vol. 64, N. ${ }^{\circ}$ 1, pp. 111-129. 
Oughton, Christine y Whittam, Geoff (1997). Competition and cooperation in the small firm sector. En: Scottish Journal of Political Economy, N. ${ }^{\circ} 44$, pp. 1-30.

Porter, Michael (1990). The Competitive Advantage of Nations. Harvard Business Review. En: Harvard Business Review, Vol. 68, N. ${ }^{\circ}$ 2, marzo-abril, pp. 73-93.

Rendón, Jaime (2009). Industrialización y dinámicas espaciales en Bogotá: las urgencias de la gestión territorial. En: Semestre Económico, Vol. 12, N.ㄹ 24, Universidad de Medellín, Medellín, pp. 39-62.

Rendón, Jaime (2012). Desarrollo, industrialización y territorio en Bogotá: Evolución productiva y desindustrialización. Editorial Académica Española (EAE), Madrid, 416 pp.

Romer, P. (1986). Incrasingreturns and long run growth. En: Journal of Political Economy, N. 94 , pp. 1002-1037.

Scitovsky, T. (1954). Two concepts if external economies. En: Journal of Political Economy, No 62, $143 \mathrm{pp}$.

Vázquez, Antonio (2000). La política del desarrollo económico local. CEPAL, 25 pp.

Vázquez, Antonio (2006). Surgimiento y transformación de clústers y milieus en los procesos de desarrollo. En: Revista Eure, No. 95, Santiago de Chile, pp. 75-92. 